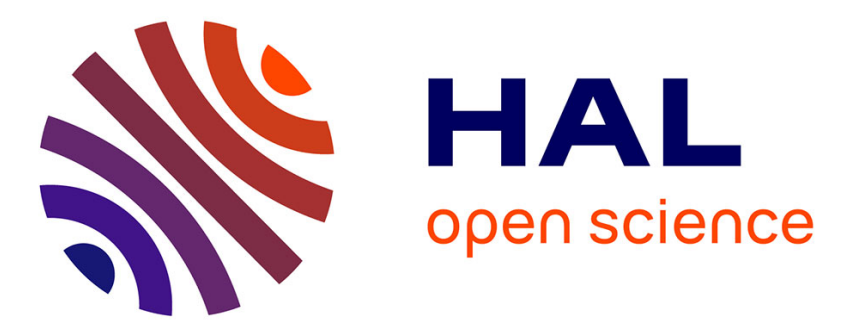

\title{
Rationally Designed Single-Crystalline Nanowire Networks
}

Diana Car, Jia Wang, Marcel A. Verheijen, Erik P.A.M. Bakkers, S.R. Plissard

\section{To cite this version:}

Diana Car, Jia Wang, Marcel A. Verheijen, Erik P.A.M. Bakkers, S.R. Plissard. Rationally Designed Single-Crystalline Nanowire Networks. Advanced Materials, 2014, 26 (28), pp.4875 - 4879. 10.1002/adma.201400924 . hal-01080109

\section{HAL Id: hal-01080109 \\ https://hal.science/hal-01080109}

Submitted on 4 Nov 2014

HAL is a multi-disciplinary open access archive for the deposit and dissemination of scientific research documents, whether they are published or not. The documents may come from teaching and research institutions in France or abroad, or from public or private research centers.
L'archive ouverte pluridisciplinaire HAL, est destinée au dépôt et à la diffusion de documents scientifiques de niveau recherche, publiés ou non, émanant des établissements d'enseignement et de recherche français ou étrangers, des laboratoires publics ou privés. 


\title{
Rationally Designed Single-Crystalline Nanowire Networks
}

\author{
Diana Car ${ }^{1, *}$, Jia Wang ${ }^{1}$, Marcel A. Verheijen ${ }^{1,2}$, Erik P.A.M. Bakkers ${ }^{1,3}$, Sébastien R.
} Plissard $^{3,4,5}$

${ }^{1}$ Department of Applied Physics, Eindhoven University of Technology, P.O. Box 513, 5600 MB Eindhoven, the Netherlands

${ }^{2}$ Philips Innovation Services Eindhoven, High Tech Campus 11, 5656AE Eindhoven, the Netherlands

${ }^{3}$ Kavli Institute of Nanoscience, Delft University of Technology, 2628CJ Delft, the Netherlands

${ }^{4}$ CNRS, LAAS, 7 avenue du Colonel Roche, F-31400 Toulouse, France

${ }^{5}$ Univ. Toulouse, LAAS, F-31400 Toulouse, France

*Electronic mail: d.car@tue.nl

KEYWORDS: Nanowires, Nanocrosses, Networks, InSb, Growth Mechanisms

Rational bottom-up assembly of nanowire networks may be a way to successfully continue the miniaturization, which is the main driving force behind the semiconductor industry. So far, the fabrication of branched nanowire structures is based on processes, which are limiting the control over crystalline quality, position of the junction and overall morphology. ${ }^{[1,2]}$ Here we report a rational bottom-up epitaxial fabrication of planar nanowire architectures. Bottom-up growth of nanowires is the preferred route as it provides complex contact schemes, such as gates underneath and superconducting contacts on top of the structure, which cannot be easily achieved by top-down methods. Furthermore, nanowire growth grants a great degree of freedom in combining materials with different lattice parameters and doping profiles, ${ }^{[3,4]}$ allowing for configurations impossible to realize in thin film geometry. 
Achieving a single crystalline nanowire junction is nevertheless a challenging task. ${ }^{[5,6]}$ Defects are undesirable as they might induce carrier scattering and therefore reduce the transport properties. ${ }^{[7]}$ We use the substrate crystallography to ensure the nanowires meet under the optimal angle for formation of single-crystalline structures. Moreover, by exact control of the position of the catalyst in two directions we can tune the type, as well as the number, of wire-to-wire junctions.

Our method is generic and can be employed to synthesize interconnected nanowire architectures of group III-V, II-VI and IV materials. InSb is our material of choice due to its superior transport properties ${ }^{[8,9]}$ which make it a promising platform for the future random access memories based on Majorana fermions (MFs). ${ }^{[10,11,12,13]}$ The challenge ahead is to design and fabricate new quantum devices, which would allow for braiding of MFs - an operation, which should bring us one step closer to topological quantum computing with these quasiparticles. The simplest circuit necessary to demonstrate the braiding of Majorana fermions consists of five superconducting islands connected by a 2-junction nanowire structure and enclosed in a transmission line resonator. ${ }^{[14]}$ To fully exploit computational power of Majoranas, i.e. to allow for multiple independent braiding operations, complex nanowire systems with more than two junctions are demanded. ${ }^{[15]}$ It is of great importance to develop a novel method of material synthesis, which would enable the controlled fabrication of planar interconnected nanowire architectures.

In order to control the nanowire position, arrays of gold islands are patterned on (001) InP substrate by electron beam lithography (EBL). InP nanowires grown on InP (001) substrates are used as stems for InSb nanowire growth to facilitate the nucleation 
of InSb wires, which is difficult on flat surfaces. ${ }^{[16,17]}$ We use an InP substrate because $P$ from the substrate cannot easily be incorporated into InSb due to the large miscibility gap in the ternary, ${ }^{[18]}$ in contrast to As when an InAs substrate is used. Hence, a vertical $<001>\operatorname{lnP}$ segment is first grown (Figure 1a). ${ }^{[19]}$ It is then possible to change the growth direction of the $\operatorname{InP}$ nanowires into a $<111>\mathrm{B}$ direction (Figure $1 \mathrm{~b}$ ) by changing the contact angle of the catalyst droplet. ${ }^{[20]}$ These kinked InP nanowires are then used as stems for the growth of InSb nanowires (Figure 1c). As already reported, ${ }^{[21,22,23]}(001)$ substrates are ideal candidates for the growth of complex structures since two $<111>B$ directions are available. To be more precise, on an (001) III-V substrate there are 4 $<111>$ directions pointing upwards of which two correspond to $<111>\mathrm{A}$ and two to $<111>B$ directions. As several studies have shown that $\ln P$ and $\ln S b$ nanowires normally grow in a $<111>\mathrm{B}$ direction, ${ }^{[24]}$ we can conclude that there are only two growth directions available on an (001) III-V substrate (Figure 1d). Hence, $50 \%$ of the InSb wires will grow in the $[11-1] \mathrm{B}$ and $50 \%$ in the $[-1-1-1] \mathrm{B}$ direction. ${ }^{[20]}$ Since the InSb wires are epitaxially connected to the substrate they will meet under an angle of $109.5^{\circ}$ (Figure 1e), which is precisely the angle between two <111>B directions in a zincblende monocrystal. This, plus the fact that InSb wires crystallize in the defect-free zincblende crystal structure (as opposed to wurtzite InAs nanowires), is why our approach is expected to result in a high yield of single-crystalline junctions.

We first discuss the situation in which the catalyst particles are exactly aligned along the [-1-10] crystalline direction of the substrate (Figure 2a). When two InSb nanowires, growing towards each other (respectively in the $[-1-1-1] \mathrm{B}$ and the $[11-1] \mathrm{B}$ directions) meet, they touch tip-to-tip. The two gold droplets coalesce into a bigger one 
and a nanowire "bridge" is formed (Figure 2b,c). ${ }^{[21]}$ Although this is an interesting approach to double the length of the InSb wires while maintaining the relatively small diameter, we are interested in multi-branched wires. In order to assist formation of crossing events, arrays with a misalignment in the y-direction were designed (Figure 2d). In this case, two InSb nanowires, growing towards each other from two mutually misaligned droplets, do not merge into a "bridge" structure. Instead, they grow in close proximity and finally merge due to radial nanowire growth forming a nanocross (Figure $2 e, f)$. The crystalline structure of the junctions is investigated by transmission electron microscopy (TEM). Figure 3a shows a bright field TEM image of a crossed junction formed by two wires meeting under an optimal angle of $109.5^{\circ}$. The high resolution TEM images taken just below the droplets (Figure $3 b, d$ ) of two merged nanowires and the corresponding Fast-Fourier Transforms (FFT) (Figure 3c,e) show that the crystalline directions of both legs are identical. This structure is therefore a single crystal. Nevertheless, the HR-TEM image of another junction (Figure S2, Supporting Information) shows a Moiré interference pattern which is a signature of two differently oriented crystals. The corresponding FFT pattern reveals a twinning diffraction pattern, with a $36.5^{\circ}$ angle between the $<200>$ directions of the two nanowires. This structure is not a single crystal, and there is a grain boundary at the junction. The formation of the grain boundary can be explained by rotational defects in the InP stem. The vertical $<001>\ln \mathrm{P}$ segment is defect-free whereas twinning occurs in the kinked $<111>\mathrm{B} \ln \mathrm{P}$ segment (Figure S4, Supporting Information). After an odd number of rotational twins is formed in the $<111>B \operatorname{InP}$ nanowire, the top of the $<111>B \operatorname{InP}$ nanowire and the (001) InP substrate are twin-related. On the contrary, if there is an even number of rotational 
twins in the $<111>B \ln P$ segment, its orientation is again identical to that of the substrate. Thus, if an even number of rotational twins is present in both the [11-1]B and $[-1-1-1] \mathrm{B} \operatorname{InP}$ stem, the resulting crystalline orientations at the end of the two $\ln P$ segments are identical. The same applies for InSb nanowires growing on top of them, and the resulting crossed junction is in that case single-crystalline.

It is important to notice that the orientation of a [11-1]B InP segment after an odd number of twins is not the same as the orientation of the $[-1-1-1] \mathrm{B} \ln \mathrm{P}$ segment after an odd number of twins, since the rotations occur around different axes. In this case, a grain boundary is formed at the junction between the two InSb nanowires. The FFT pattern of such a twinned-twin junction will show a $36.5^{\circ}$ angle between the two $<200>$ directions, as shown in Figure S2, Supporting Information. Finally, an InSb nanocross can be formed from two InSb nanowires, one growing from an InP stem with an even number of twins, and one from an InP stem with an odd number of twins. The FFT diffraction pattern of such a twinned junction will show a $109.5^{\circ}$ angle between the two $<200>$ directions (Figure S3, Supporting Information). Taking into account all the possible crystalline orientations of $\operatorname{InP}$ nanowires, and the fact that there is a $50 \%$ chance for an even and odd number of twin boundaries in an InP segment, we are left with four possible options: even-even, odd-odd, and even-odd/odd-even (Figure S5, Supporting Information); each of which has a $25 \%$ probability.

We note that with this method the yield of single crystalline junctions has been increased significantly compared with our previously reported approach (from $8 \%$ to $25 \%),{ }^{[25]}$ but further improvements are desirable for efficient device fabrication. One possible way to further increase the yield of single-crystalline nanostructures is to use 
defect-free InP nanowires as stems for InSb nanowire growth. In this manner we would be able to increase the yield of perfectly single-crystalline structures from $25 \%$ to $100 \%$. For this the growth of defect-free zinc-blende $<111>B \ln P$ wires has to be developed, which has not been reported so far. An alternative route is to directly grow InSb nanowires on the vertical $<001>\mathrm{InP}$ nanowires, which are defect-free (Figure S4, Supporting Information). It is, however, difficult to nucleate InSb wires on top of these stems because during the cool down of the $<001>\operatorname{lnP}$ nanowires a defect-free zincblende $<111>B$ segment is formed. ${ }^{[20]}$ The low surface energy of the AulnSb droplet on top of the kinked $<111>B$ segment causes the wetting of the $\operatorname{InP}$ nanowire sidewalls, which will prevent InSb nanowires from nucleating.

A significant advantage of our method is the possibility of controlled fabrication of planar multiple wire structures. Important to mention is that the type of structure, which is predominantly formed, can be tuned by the catalyst spacing and offset. Figure $4 a$ illustrates what we denote as a $\pi$-structure, as its shape resembles the one of the greek letter $\pi$. It is formed out of 3 wires, 2 of which are growing from mutually aligned catalyst particles and are therefore forming a nanowire-bridge structure, while the $3^{\text {rd }}$ nanowire is misaligned with respect to the other two and is crossing one of the two legs of a bridge. Figure 4b shows a slightly different $\pi-$ structure, consisting of 3 wires and 2 crossed junctions. An example of a single-crystalline $\pi$ - junction is shown in the Figure S1, Supporting Information, showing that this approach is suitable for fabricating key components of future Majorana random access memories.

4 nanowires can be merged in a way depicted in Figure 4c - two nano-bridges connected by one crossed junction - or they can form a closed nanowire loop as shown 
in Figure 4d. Bottom up synthesis of closed nanowire loops is an entirely novel concept. A nanowire loop is formed out of four interconnected InSb nanowires, two of which are growing in the [11-1]B and the other two in [-1-1-1]B direction. In order to synthesize such complex structures one needs to be able to grow high yield $<001>\ln P$ nanowires, exactly control the $<001>$ to $<111>B$ InP kinking event and be able to grow high aspect ratio $\mathrm{InSb}$ nanowires which should be at least $2.5 \mu \mathrm{m}$ long in order to promote a double crossing event, necessary for the loop formation. Key concept here is again the misalignment of the gold droplets in an array, which allows us to grow crossed nanowire structures instead of nanowire-bridges. Our InSb nanowire loops are a promising material system, not only as building blocks of future Majorana random access memories, but also for exciting new physics experiments on coherent transport through the nanostructures from which the phase coherence length of electrons in $\operatorname{lnSb}$ nanowires may be extracted.

Figure 4e illustrates a 6-wire structure: a closed 4 - nanowire loop whose one leg is forming a $\pi$-structure with the remaining 2 nanowires. A network formed out of 7 interconnected wires is shown in Figure 4f. Improvements in the length, as well as in the yield of InSb nanowires on (001) substrate, would allow for controllable growth of even larger InSb nanowire networks. Furthermore, the method presented is general, such that it can be applied to assemble complex nanowire structures of other group III-V, II-VI and IV semiconductor materials and even hetero materials.

To summarize, we demonstrate an unprecedented level of control in growth of complex InSb nanowire structures. We report, for the first time, planar, crystalline nanowire structures formed out of more than two nanowires - nanowire $\pi$-structures, 
closed nanowire loops and nanowire networks The perfect control of the droplet positioning, i.e. the offset between the neighboring gold droplets in an array, allows us to favor crossed junctions over "bridge"-junctions and, in such a way, increase the yield of advantageous structures.

\section{Experimental Section}

Nanowire growth: Nanowires have been grown on (001) InP substrate using Aucatalyzed vapor-liquid-solid process by metal organic vapor phase epitaxy in an Aixtron MOVPE machine. InP $<001>$ nanowires were first grown at a temperature of $440{ }^{\circ} \mathrm{C}$ for 9 min using tri-methyl-indium (TMI) and phosphine $\left(\mathrm{PH}_{3}\right)$ as precursors with growth pressure of 50 mbar and precursor molar fractions $X i(\mathrm{TMI})=2.42 \times 10^{-5}$ and $X i\left(\mathrm{PH}_{3}\right)=$ $7.44 \times 10^{-3}$. To increase the yield of vertical wires growing in the $<001>$ direction the $\mathrm{Au}$ droplet shape and composition was optimized prior to wire growth: when the system reached the temperature of $440{ }^{\circ} \mathrm{C}$ under $\mathrm{PH}_{3}$ pressure, we simultaneously switched $\mathrm{PH}_{3}$ off and TMI on for $15 \mathrm{~s}$. These high yield $<001>\operatorname{InP}$ nanowires are used as templates for switching of the growth direction. After $9 \min$ of $<001>$ nanowire growth, TMI is switched off for 2 min while $\mathrm{PH}_{3}$ is kept on. When we re-start the growth by switching TMI on again, nanowires grow in the $<111>B$ direction. $\ln P<111>B$ segments are grown using the same temperature and precursor flows as the $<001>\ln P$ wires.

These kinked InP nanowires are then taken out of the growth reactor and transported, in nitrogen atmosphere, to the other Aixtron MOVPE machine dedicated to the growth of antimonides only. InSb nanowires are grown on top of the kinked InP stems at $495{ }^{\circ} \mathrm{C}$ 
for $20 \mathrm{~min}$, using tri-methyl-indium (TMI) $\left(X i=1.1 \times 10^{-5}\right)$ and tri-methyl-antimony (TMSb) $\left(X i=3.4 \times 10^{-3}\right)$.

\section{Supporting Information}

Supporting Information is available from the Wiley Online Library or from the author.

\section{Acknowledgements}

This work has been supported by the Dutch Organization for Scientific Research (NWO), the Foundation for Fundamental Research on Matter (FOM) and the European Union Seventh Framework Programme under grant agreement no. 265073.

\section{Additional information}

The authors declare no competing financial interests. 


\section{References}

(1) X. Jiang, B. Tian, J. Xiang, F. Qian, G. Zheng, H. Wang, L. Mai, C. M. Lieber, PNAS 2011, 108, 12212.

(2) D. Gedamu, I. Paulowicz, S. Kaps, O. Lupan, S. Wille, G. Haidarschin, Y. K. Mishra, R. Adelung, Adv. Mater., DOI: 10.1002/adma.201304363.

(3) B. - M. Nguyen, Y. Taur, S. T. Picraux, S. A. Dayeh, Nano Lett. 2014, 14, 585.

(4) A. W. Dey, M. Borg, B. Ganjipour, M. Ek, K. A. Dick, E. Lind, C. Thelander, L. - E. Wernersson, IEEE Electron device letters 2013, 34, 211.

(5) S. R. Plissard, D. R. Slapak, M. A. Verheijen, M. Hocevar, G. W. G. Immink, I. van Weperen, S. Nadj-Perge, S. M. Frolov, L. P. Kouwenhoven, E. P. A. M. Bakkers, Nano Lett. 2012, 12, 1794.

(6) M. Jeppsson, K. A. Dick, J. B. Wagner, P. Caroff, K. Deppert, L. Samuelson, L. - E. Wernersson, J. Crystal Growth 2008, 310, 4115.

(7) C. Thelander, P. Caroff, S. R. Plissard, A. W. Dey, K. A. Dick, Nano Lett. 2011, 11, 2424.

(8) J. M. S. Orr, A. M. Gilbertson, M. Fearn, O. W. Croad, C. J. Storey, L. Buckle, M. T. Emeny, P. D. Buckle, T. Ashley, Phys. Rev. B 2008, 77, 165334.

(9) R. L. Kallaher, J. J. Heremans, N. Goel, S. J. Chung, M. B. Santos, Phys. Rev. B 2010, 81, 75303. 
(10) E. Majorana, Soryushiron Kenkyu (Engl. transl.) 1981, 63, 149 [transl. from Teoria simmetrica dell' elettrone e del positrone, Nuovo Cimento 1937, 14, 171].

(11) V. Mourik, K. Zuo, S. M. Frolov, S. R. Plissard, E. P. A. M. Bakkers, L. P. Kouwenhoven, Science 2012, 336, 1003.

(12) M. T. Deng, C. L. Yu, G. Y. Huang, M. Larsson, P. Caroff, H. Q. Xu, Nano Lett. 2012, 12, 6414 .

(13) A. Das, Y. Ronen, Y. Most, Y. Oreg, M. Heiblum, H. Shtrikman, Nat. Phys. 2012, 8, 887.

(14) T. Hyart, B. van Heck, I. C. Fulga, M. Burrello, A. R. Akhmerov, C. W. J. Beenakker, Phys. Rev. B 2013, 88, 35121.

(15) J. Alicea, Y. Oreg, G. Refael, F. von Oppen, M. P. A. Fisher, Nat. Phys. 2011, 7, 412.

(16) P. Caroff, J. B. Wagner, K. A. Dick, H. A. Nilsson, M. Jeppsson, K. Deppert, L. Samuelson, L. R. Wallenberg, L. E. Wernersson, Small 2008, 4, 878.

(17) B. Mandl, K. A. Dick, D. Kriegner, M. Keplinger, G. Bauer, J. Stangl, K. Deppert, Nanotech. 2011, 22, 145603.

(18) M. Heuken, C. V. Eichel-Streiber, A. Behres, B. Schineller, K. Heime, C. Mendorf, G. Brockt, H. Lakner, J. Electron. Mat. 1997, 26, 1221.

(19) J. Wang, S. R. Plissard, M. Hocevar, T. T. T. Vu, T. Zehender, G. W. G. Immink, M. A. Verheijen, J. Haverkort, E. P. A. M. Bakkers, Appl. Phys. Lett. 2012, 100, 053107. 
(20) J. Wang, S. R. Plissard, M. A. Verheijen, L. F. Feiner, A. Cavalli, E. P. A. M. Bakkers, Nano Lett. 2013, 13, 3802.

(21) D. Dalacu, A. Kam, D. G. Austing, P. J. Poole, Nano Lett. 2013, 13, 2676.

(22) S. Conesa-Boj, E. Russo-Averchi, A. Dalmau-Mallorqui, J. Trevino, E. F. Pecora, C. Forestiere, A. Handin, M. Ek, L. Zweifel, L. R. Wallenberg, D. Rüffer, M. Heiss, D. Troadec, L. Dal Negro, P. Caroff, A. Fontcuberta i Morral, ACS Nano 2012, 6, 10982.

(23) J. H. Kang, Y. Cohen, Y. Ronen, M. Heiblum, R. Buczko, P. Kacman, R. PopovitzBiro, H. Shtrikman, Nano Lett. 2013, 13, 5190.

(24) H. A. Fonseka, H. H. Tan, J. Wong-Leung, J. H. Kang, P. Parkinson, C. Jagadish, Nanotech. 2013, 24, 465602.

(25) S. R. Plissard, I. van Weperen, D. Car, M. A. Verheijen, G. W. G. Immink, J. Kammhuber, L. J. Cornelissen, D. B. Szombati, A. Geresdi, S. M. Frolov, L. P. Kouwenhoven, E. P. A. M. Bakkers, Nature Nanotech. 2013, 8, 859. 

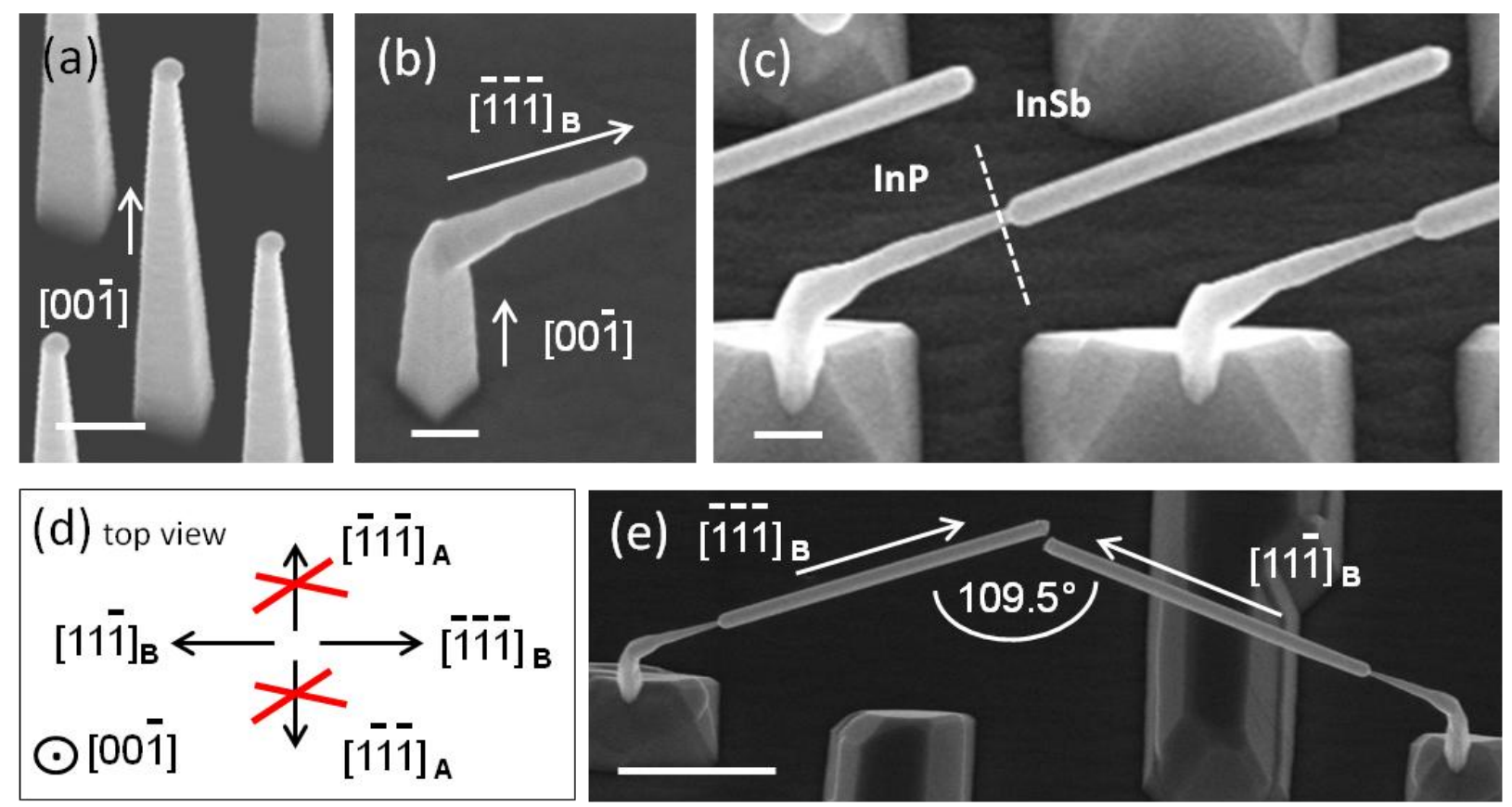

Figure 1. InSb nanowires grown on an (001) InP substrate. (a) $<001>\ln P$ nanowires grown on an (001) InP substrate. (b) A kinked <111>B InP segment on top of the vertical $<001>\operatorname{InP}$ nanowire. (c) High aspect ratio <111>B InSb nanowires grown on kinked InP stems in (b). (d) A scheme indicating four $<111>$ directions pointing upwards from (001) substrate. InSb nanowires only grow in $<111>B$ direction. (e) Two InSb nanowires growing at an angle of $109.5^{\circ}$ with respect to each other. For images (a), (b) and (c) the scale bar is $200 \mathrm{~nm}$. For image (e) the scale bar is $1 \mu \mathrm{m}$. For all SEM images the viewing angle is $30^{\circ}$. 

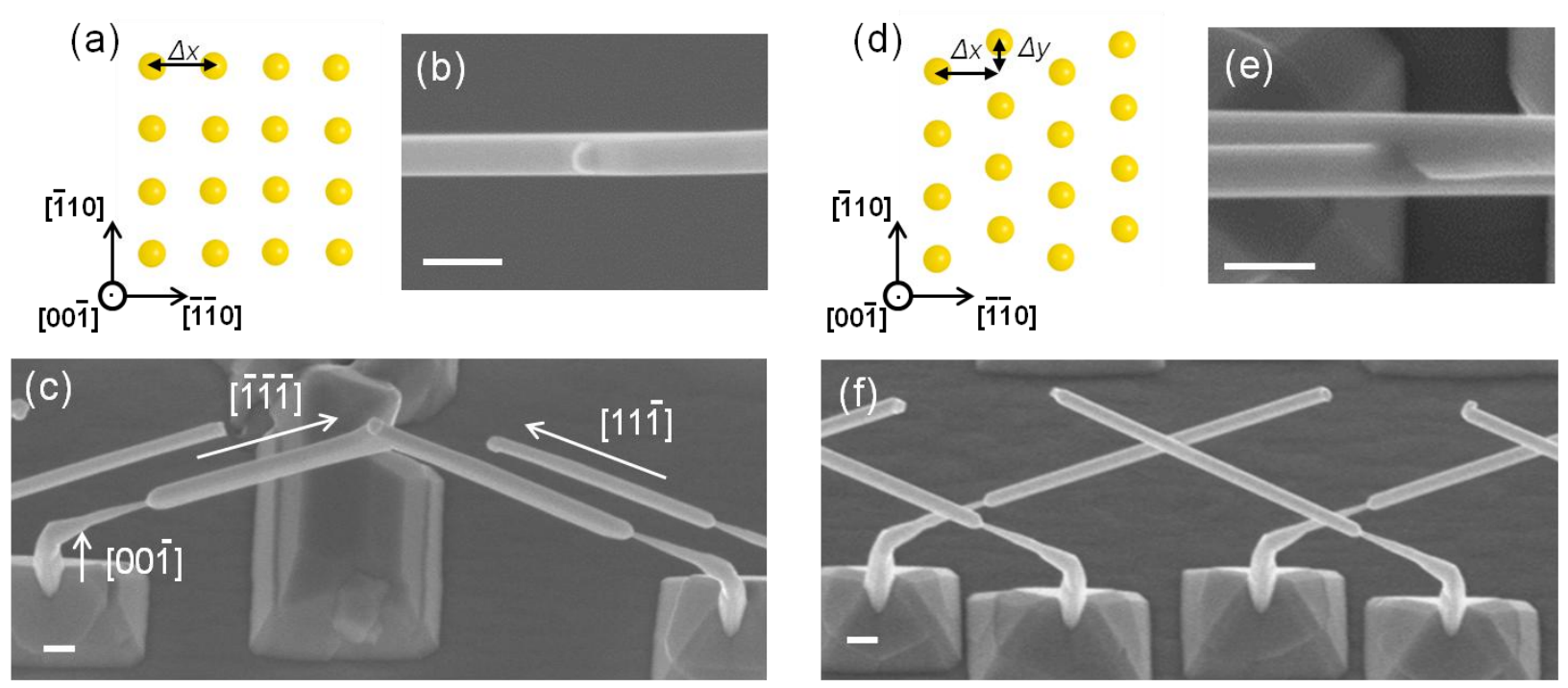

Figure 2. Position - and direction - controlled growth of InSb nanowire networks.

(a) Scheme of a gold droplet array defined by EBL. $\Delta x$ denotes the pitch between the two neighboring droplets. (b) Top-view SEM image of two merged nanowires. A single gold droplet on top of the nanowire-bridge structure can be easily seen. (c) $30^{\circ}$ tilted SEM image of the complete bridge structure (kinked InP stems + merged InSb nanowires) with growth directions indicated by arrows. (d) Introducing the misaligned EBL array of gold catalysts to favor crossed nanowire structures. $\Delta x$ denotes the pitch and $\Delta y$ the offset between the two neighboring droplets. (e) Top-view SEM image showing an InSb crossed junction. (f) $30^{\circ}$ tilted SEM image of the crossed structure. All scale bars correspond to $200 \mathrm{~nm}$. 

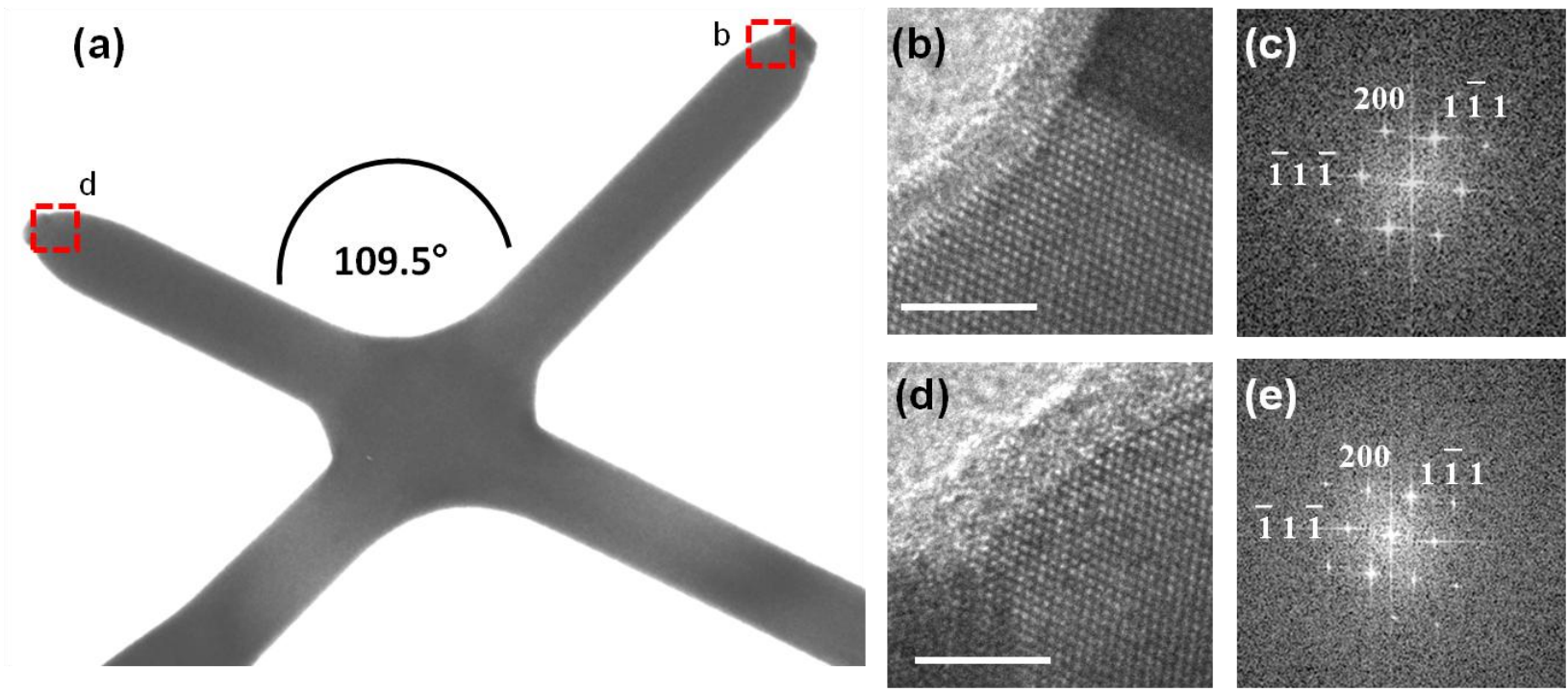

Figure 3. Transmission Electron Microscopy (TEM) study of a single-crystalline junction. (a) Bright Field TEM image of a single-crystalline junction. (b) and (d) are HRTEM images taken just below the droplets of each leg. (c) and (e) are the corresponding FFT patterns. The crystalline directions in (c) and (e) are perfectly superposed, which proves the single-crystallinity of the junction. 

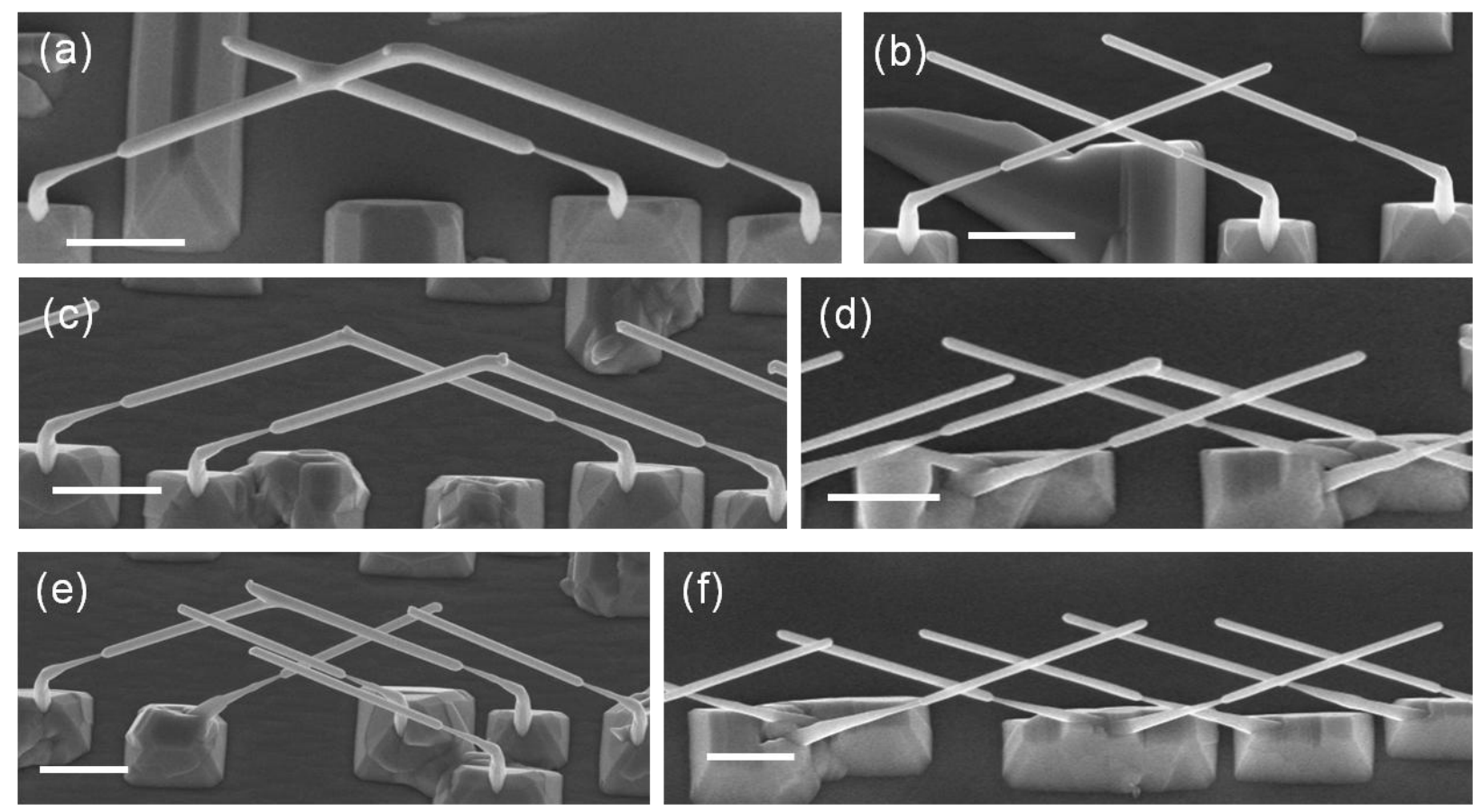

Figure 4. Nanowire networks. (a) $30^{\circ}$ tilted SEM image of the nanowire $\pi-$ structure. Two nanowires growing from mutually aligned droplets merge into a bridge. A third nanowire growing from a misaligned droplet forms a crossed junction with one leg of the bridge. (b) $30^{\circ}$ tilted SEM image of the nanowire $\pi$ - structure consisting of three nanowires and two crossed junctions. (c) $30^{\circ}$ tilted SEM image of a 4-nanowire architecture: two nanowire bridges connected by one crossed junction. (d) $30^{\circ}$ tilted SEM image of the nanowire loop formed out of four interconnected InSb nanowires, two of which are growing in the $[11-1] \mathrm{B}$ and the other two in the $[-1-1-1] \mathrm{B}$ direction. (e) $30^{\circ}$ tilted SEM image of the 6 - nanowire network: 4 nanowires connected in a closed loop whose one leg is forming a $\pi$-structure with the other 2 nanowires. (f) $30^{\circ}$ tilted SEM image of a 7-nanowire network. All scale bars $1 \mu \mathrm{m}$. 
Copyright WILEY-VCH Verlag GmbH \& Co. KGaA, 69469 Weinheim, Germany, 2013.

\section{Supporting Information}

\section{Rationally designed single-crystalline nanowire networks}

Diana Car ${ }^{1, *}$, Jia Wang ${ }^{1}$, Marcel A. Verheijen ${ }^{1,2}$, Erik P.A.M. Bakkers ${ }^{1,3}$, Sébastien R. Plissard ${ }^{3,4,5}$

Summary of the supplementary materials:

Section 1: TEM study of a single-crystalline $\pi-$ structure.

Section 2: TEM study of a twinned-twin junction.

Section 3: TEM study of a twinned junction.

Section 4: Bright field TEM of an InP + InSb nanowire structure.

Section 5: A table summarizing all possible crossing scenarios.

Section 6: Formation of InSb bridge structures. 
Section 1: TEM study of a single-crystalline $\pi-$ structure.
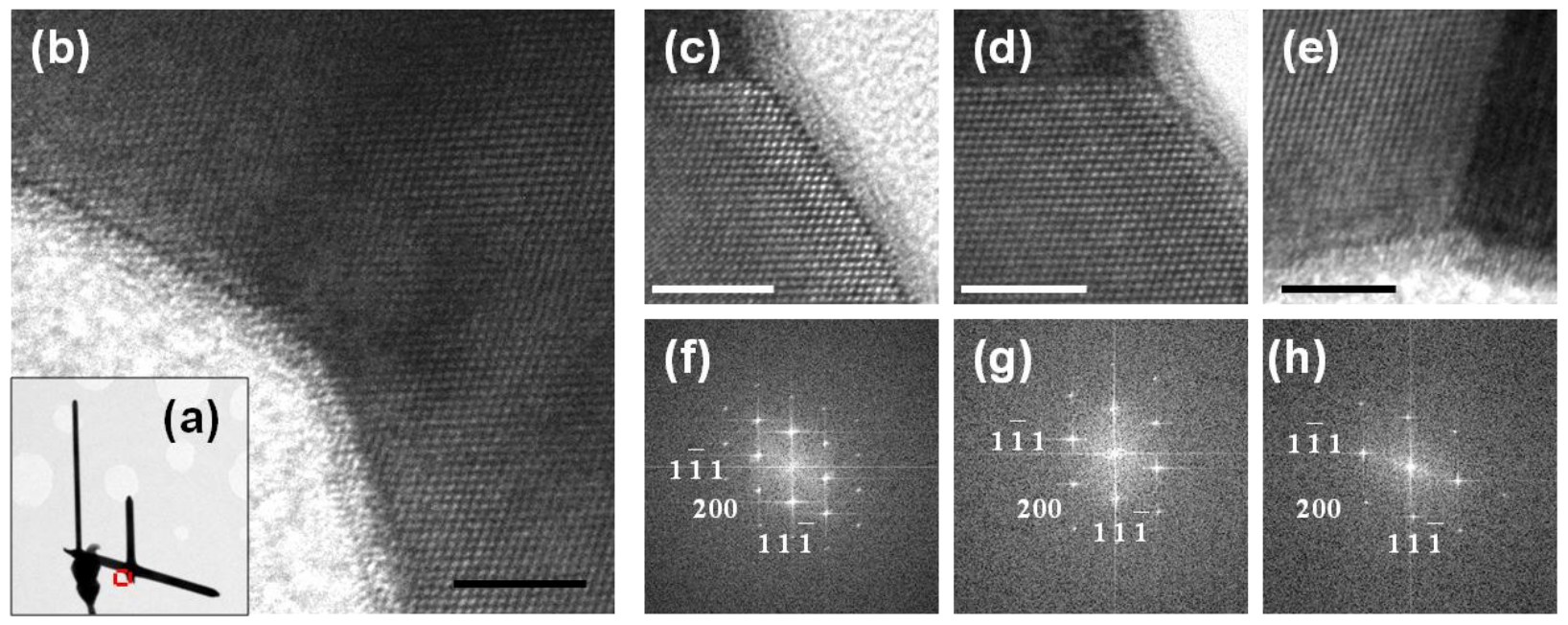

Figure S1: Single-crystalline InSb $\pi$ - structure.

(a) Bright field TEM image of a single - crystalline nanowire $-\pi$. One leg of a structure is broken off during the transfer to the TEM grid. Another InSb nanowire is partially covering one junction of the $\pi$. (b) High resolution TEM image taken at a junction (indicated by red square in (a)). Scale bar $5 \mathrm{~nm}$. (c), (d) and (e) are HRTEM images taken in the regions right under the gold droplets of the three nanowires forming a $\pi$. All scale bars $5 \mathrm{~nm}$. (f), (g) and (h) are FFTs corresponding to (c), (d) and (e), respectively. All three FFTs are a perfect match indicating that three merged nanowires share the same crystalline orientation and the resulting $\pi$ structure is a single crystal. 
Section 2: TEM study of a twinned-twin crossed junction
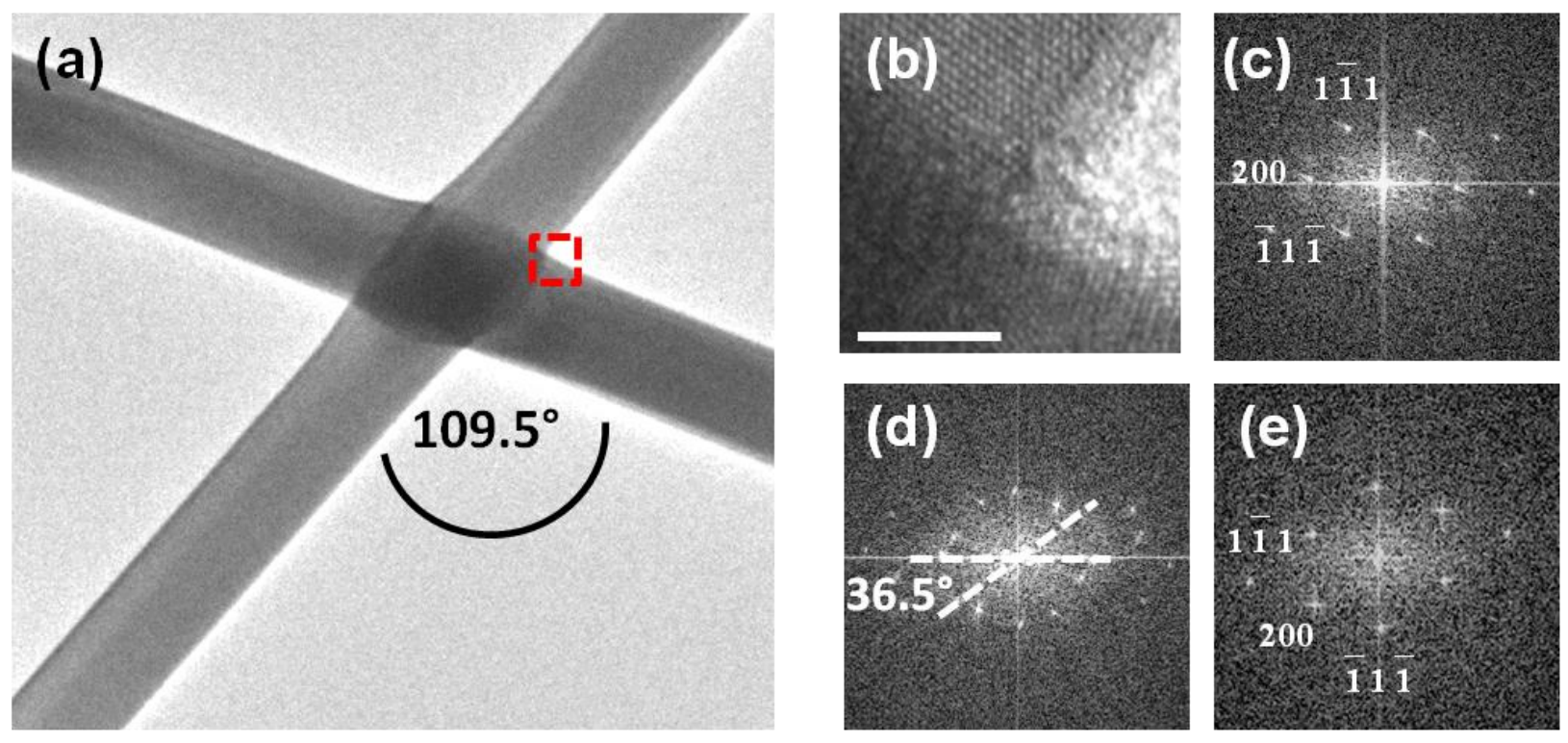

Figure S2: InSb nanocross with a twinned-twin junction.

(a) Bright field TEM image of a nanocross with an optimal meeting angle of $109.5^{\circ}$.

(b) High resolution TEM image taken at a junction (indicated by red square in (a)). Moiré pattern can be seen. Scale bar $5 \mathrm{~nm}$.

(c) and (e) are FFTs of two nanowires forming a cross. It can be seen that the crystalline orientation of the two nanowires is not the same.

(d) FFT corresponding to HRTEM image in (b). There is a twinned-twin relation between crystallographic orientations of the two nanowires, the angle between the two $<200>$ directions being $36.5^{\circ}$. 
Section 3: TEM study of a twinned T-structure.
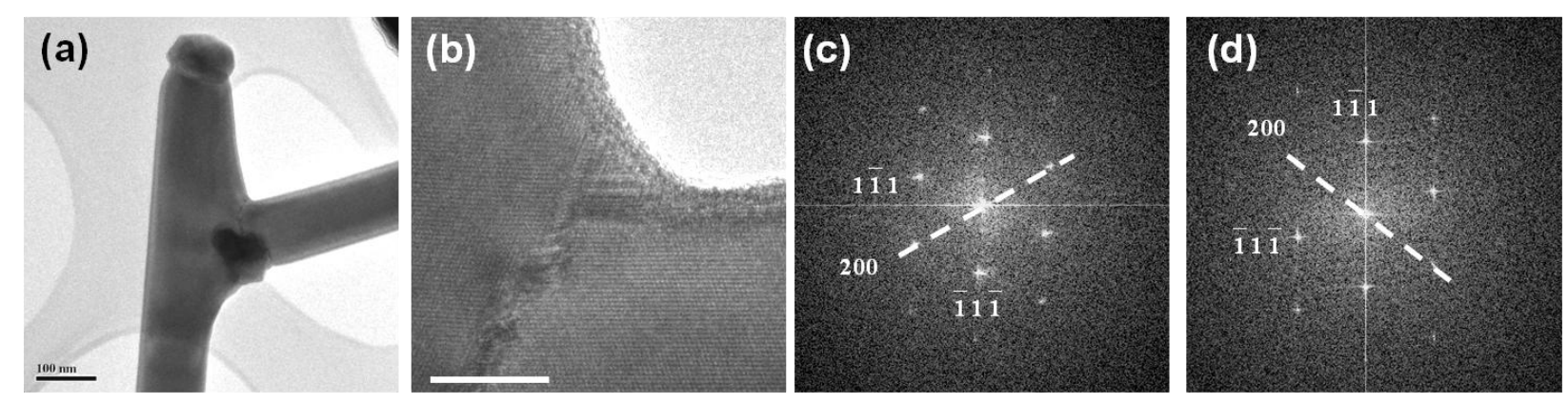

Figure S3: InSb T-structure with a twinned junction.

(a) Bright field TEM image of a nanowire $\mathrm{T}$ - structure containing a single grain boundary at a junction. Scale bar $100 \mathrm{~nm}$.

(b) HRTEM image taken at a junction. The grain boundary can be seen. Scale bar 10 $\mathrm{nm}$.

(c) and (d) are FFT diffraction patterns of two nanowires forming a T-structure. The crystalline orientations of the two nanowires do not match. The angle between the $<200>$ directions of the two nanowires (indicated by white dashed lines) is $109.5^{\circ}$. 
Section 4: Bright field TEM of an InP + InSb nanowire structure.

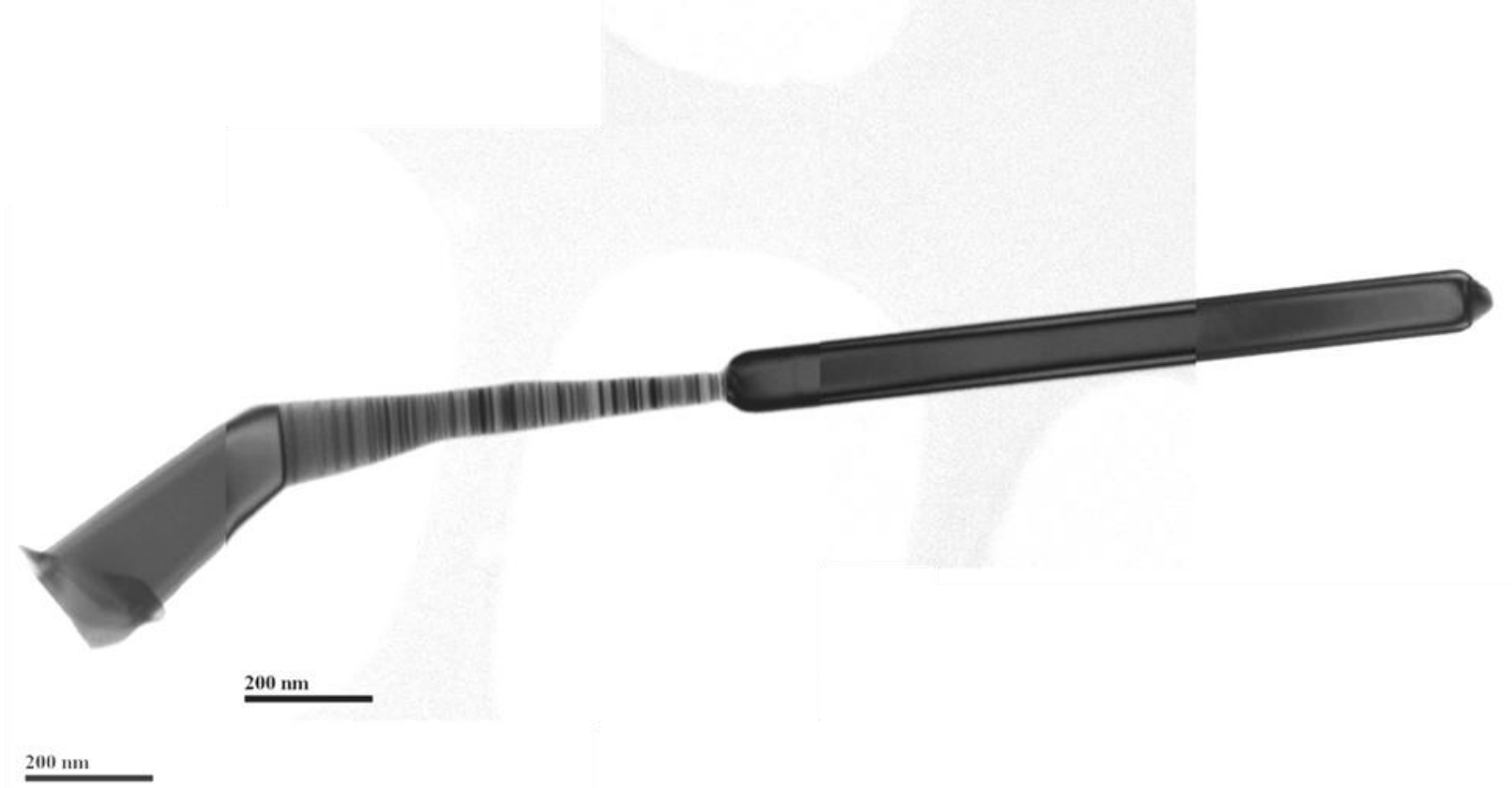

Figure S4: Bright field TEM of an InP - InSb nanowire.

From the bright field TEM image we can see that the $<001>\operatorname{InP}$ segment and $<111>$ InSb nanowire are defect free. On the contrary, a significant number of stacking faults present in the $<111>\operatorname{InP}$ segment makes it safe to conclude that there is a $50: 50$ chance an odd or even number of twin domains will be present in this segment. 
Section 5: A table summarizing all possible crossing scenarios.

\begin{tabular}{|c|c|c|c|c|}
\hline & & Junction & $\begin{array}{c}\text { Angle between <200> } \\
\text { directions }\end{array}$ & Probability \\
\hline \multirow{2ynn}{*}{ even } & even & Single Crystal & $0^{\circ}$ & $25 \%$ \\
\hline \multirow{2}{*}{ odd } & even & Twinned & $109.5^{\circ}$ & \multirow{2}{*}{$50 \%$} \\
\hline \multirow{2}{*}{ even } & odd & & & \\
\hline odd & odd & Twinned twin & $36.5^{\circ}$ & $25 \%$ \\
\hline
\end{tabular}

Table S5: Table summarizing the four possible crossing scenarios.

In $25 \%$ of the cases, the nanostructure will be single crystalline. A defect-free pure zinc blende (111) InP segment would lead to a $100 \%$ yield of single crystalline junctions. 


\section{Section 6: Formation of InSb bridge structures.}
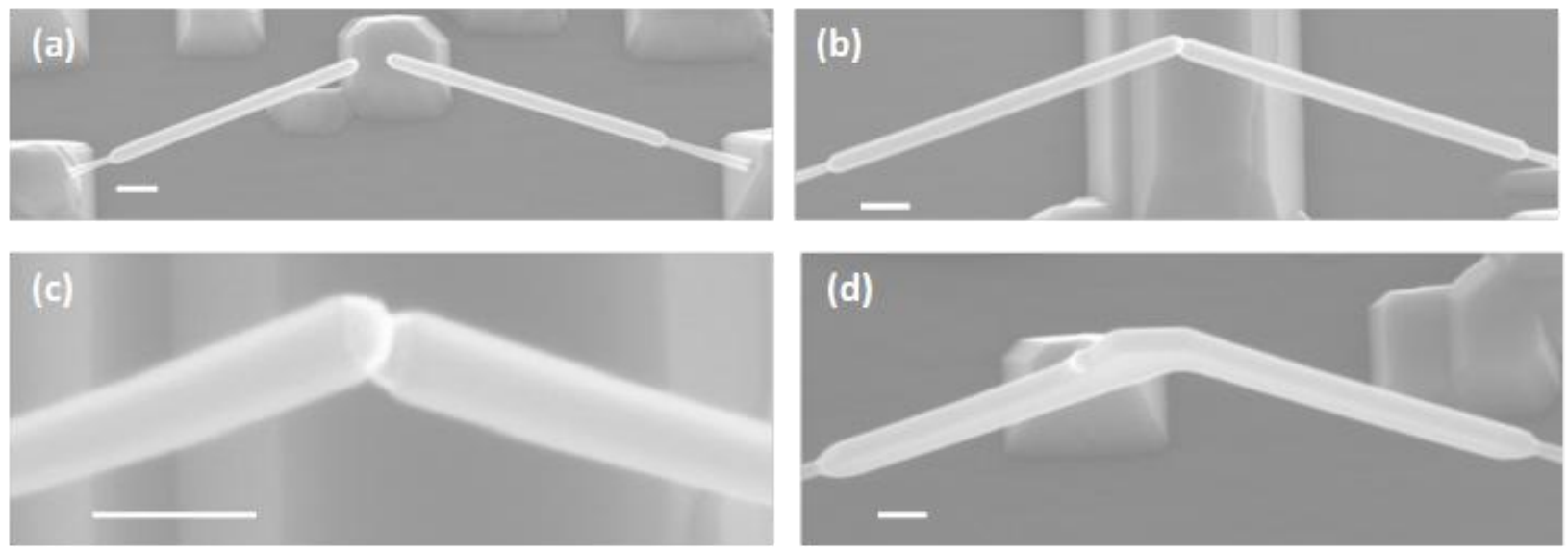

(a) Two InSb nanowires growing towards each other from two perfectly aligned, EBL defined Au islands on an (001) InP substrate.

(b) Two InSb nanowires touching tip-to-tip.

(c) High magnification SEM image of two gold droplets on top of InSb nanowires. When the gold droplets come in contact, they merge into one big droplet.

(d) The resulting InSb nanowire - bridge structure. Gold droplet is sliding along the top (110) facet of InSb nano-bridge. 